\title{
Subject Index, Vol. 70, 1995
}

This index does not contain items of the reports of the Chromosome Mapping Workshops 13 and 21 which are published in this volume.

Animals

cattle 112,192

chimpanzee 88

Chinese hamster 95

Damaliscus dorcas phillipsi (blesbok) 268

Gymnophthalmus (lizard) 29

hamster 41

mouse $48,55,143,200,263,273$

nonhuman primates 239

rat 55, 92, 263

Salvelinus namaycush (lake trout) 104

sheep 112,116

swine 186

Tupaia belangeri 258

Banded chromosome analysis blesbok (R) 268 cattle (R) 192

Gymnophthalmus (C, R, Ag-NOR) 29 human sperm (G) 80 human (DAPI) 26, 48, 64, 102, 129,183 ,

246,263 human (G) 228 human (Q) 55,218 human (R) 23,52,68,119, 195,211,215, 235 lake trout (CMA3) 104 mouse (Q) 55

nonhuman primates $(\mathrm{Q}) 239 \operatorname{rat}(\mathrm{DAPI}) 92,263 \operatorname{rat}(\mathrm{G}) 92 \operatorname{rat}(\mathrm{Q}) 55$

sable antelope (R) 268 sheep $(G) 116$ swine $(G) 224$ swine $(R) 186$

Chromosome aberration

lossofY $1 / 8$ HD 243

ring chromosome 15 and breakpoint delineation 228

sperm-chromosome abnormalities in reciprocal translocation carriers 80

spontaneous rearrangements in Chinese hamster cell lines 95

trisomy 18205

XOmice 273

Chromosome mapping workshops chromosome 13 report 1 chromosome 21 report 147

Chromosome flow sorting Chinese hamster 95

Comparative gene mapping cattle 112,192 chimpanzee 88 mouse 48,55,143,200,263

nonhuman primates 239 rat 55, 92,263 sheep 112,116 swine 186,224 tupaias 258

Fluorescent in situ hybridization (FISH) Chinese hamster chromosome specific

libraries 95 chromosome painting in oocytes of trisomy

18205 double-CMA3 chromosomes, lake trout 104 FICTION 243 microclone mapping

35,92 micronuclei in two-cell hybrid embryos 41 simple and double PRINS 138 telomeres 88 
translocation breakpoints 58 tri- and tetranucleotide repeat mapping 102 two-color FISH $129,183,258$

Gene mapping cattle

WT 112

ZNF164 192 chimpanzee

subtelomeric repeat 88 human

3p23-> p21.3cosmids 129

5SrRNAgenes 76

AHNAK 218

AHSG, HRG, KNG 26

AMD1.AMD2 195

brain ESTs 71

CALCR 246

CD2, NGFB, NRAS 183

CD24 119

CEBPD,FGFRl 188

cosmid of subtelomeric repeat 88

cosmids containing tri- and tetranucleotide repeats 102

CTNNB1 68

D3S3 134

DNASE1 221

EPAG 126

FLT3 255

GFI1 263

GLCLC 278

GNRHR 211

GPRK5, GPRK6 250

GTF3A 235

IL6ST 64

KCNA5 280

KDR 145

PTPRZ 52

SSR2 215

STD 45

STSsonllq13.4 $\rightarrow$ q25 108

TIAM1 48

YACs on chromosome 123 mouse

Alk 143

Gfil 263

Odfl 200

Tiam 148 nonhuman primates

IGHE 239 rat

Gfil 263

microclones in $\mathrm{RC}$ region 92 sheep

NRAMP 116

WT 112 
swine

FSHB, LHB 224

ORM1 186 tupaia

CENP-B box sequences 258

Genetic damage

radiation on human germ cells 41

Human disorders

growth failure and gene dosage of IGF1R

228 Hodgkin's disease (HD) 243 Renal carcinoma (RC) 92 ring chromosome 15 syndrome

228 schizophrenia 35

small cell lung carcinoma (SCLC) 119 synovial sarcoma 58 Williams syndrome (WS) 246

Wilms tumor (WT) 112 X-linked disorders 126

Hybrids

hamster-bovine 112 radiation hybrids 35 rat-mouse 92

Interspecific backcross mapping mouse 48, 143,263

Karyotype

Alcelaphinae 268 Gymnophthalmus 29

Linkage

WT region in sheep and cattle 112

Meiosis

oogenesis in human trisomy 18205 reciprocal translocation carriers 80

Microdissection and microcloning Ilq13.4 $\rightarrow \mathrm{q} 25108 \mathrm{RC}$ region in rat $92 \mathrm{t}(1,11)(\mathrm{q} 42.2 ; \mathrm{q} 21) 35$

Physical mapping 3p23 $\rightarrow$ p21.3 129

Sequence

bovine ZNF164 192

canine SSR2 215

human AMD 1 and AMD2 195

human GTF3A 235

human SSR2 215

mouse Odfl 200

STSs

human chromosome 11108

Techniques

micronucleus test using FISH 41 PRINS for specific chromosome identification 138

FICTION for analysis of complex karyotyes in HD 243

286

Cytogenet Cell Genet, Vol. 70, 1995 